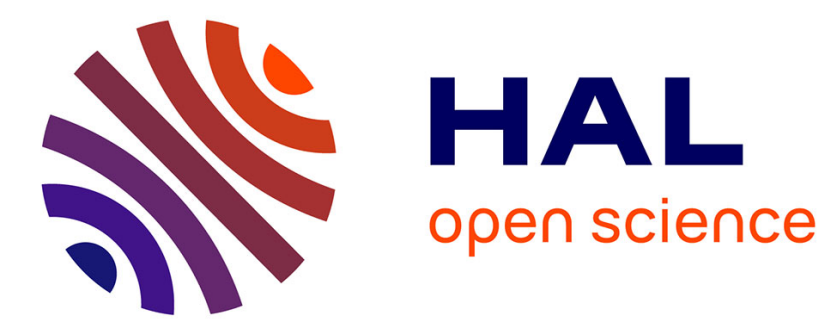

\title{
Wifi Channel Selection Based on Load Criteria
}

Danielle Saliba, Rodrigue Imad, Sébastien Houcke

\section{To cite this version:}

Danielle Saliba, Rodrigue Imad, Sébastien Houcke. Wifi Channel Selection Based on Load Criteria. WPMC 2017: 20th Internation Symposium on Wireless Personal Multimedia Communications, Dec 2017, Bali, Indonesia. 10.1109/WPMC.2017.8301833 . hal-01845233

\section{HAL Id: hal-01845233 \\ https://hal.science/hal-01845233}

Submitted on $20 \mathrm{Jul} 2018$

HAL is a multi-disciplinary open access archive for the deposit and dissemination of scientific research documents, whether they are published or not. The documents may come from teaching and research institutions in France or abroad, or from public or private research centers.
L'archive ouverte pluridisciplinaire HAL, est destinée au dépôt et à la diffusion de documents scientifiques de niveau recherche, publiés ou non, émanant des établissements d'enseignement et de recherche français ou étrangers, des laboratoires publics ou privés. 


\section{Wifi Channel Selection Based on Load Criteria}

\author{
Danielle Saliba \\ Signal and Communication Department \\ Mines Telecom Atlantique Institute \\ Brest, France \\ danielle.saliba@imt-atlantique.fr
}

\author{
Rodrigue Imad \\ Mechatronics Department \\ University of Balamand \\ Al Kurah, Lebanon \\ rodrigue.Imad@balamand.edu.lb
}

\author{
Sebastien Houcke \\ Signal and Communication Department \\ Mines Telecom Atlantique Institute \\ Brest, France \\ sebastien.houcke@imt-atlantique.fr
}

\begin{abstract}
With the increasing demand for wireless communication systems, while Heterogeneous Networks (Het-Nets) are under study toward $5 \mathrm{G}$ technology in mobile communication systems, WiFi Access Points (APs) are considered a potential layer within those multiple Radio Access Technologies (RATs). Significant network capacity gain can be achieved not only through aggressive reuse of spectrum across the multiple tiers in the network, but also by integrating WiFi in the mobile network through additional spectrum in un-licensed bands. Different criteria should be investigated in order to allow both the WiFi APs and the end user to operate on the best suitable channel, where the basic one of those criteria is the "load" of the operating channels.

We propose in this paper an accurate algorithm for the estimation of WiFi 802.11n physical channels load through 3 channels observations only. Once the channel load is estimated using the proposed algorithm, the channel assignment based on the minimal load value is acquired, thus providing faster response of an AP channel selection and faster end user connection for better Quality of Experience (QoE).
\end{abstract}

Index Terms-WiFi Channel selection, 802.11n physical layer, Power Spectral Density, Heterogeneous Networks

\section{INTRODUCTION}

With the increasing demand on wireless data communication, the main key role is focused on effective bandwidth availability given that the spectrum is limited. This issue stimulates researchers and engineers to use the spectrum more efficiently. One of the challenges faced in WiFi systems, is the channel assignment for the end user within a minimum response time from the suitable access point.

Many research studies were proposed for the channel assignment based on different criteria such as resource allocation by taking into consideration the cooperative transmission strategy [1], the power control of overlapping and nonoverlapping channels [2], or by considering the interference of clients individually [3], or by the interference relationship among clients [4], or by measurements on the Medium Access Control (MAC) layer [5], or based on scanning performance parameters leading to a minimum latency [6], ...etc.

In this paper, we propose a new algorithm that estimates the load of the WiFi 802.11n physical layer channels. Note that by channel load, we mean the percentage of the channel usage in time (or busy time) with respect to the total channel measurement time (total busy and idle time). Having the load of each channel, facilitates the decision of the user for the channel selection based on the minimal load criteria. We are simulating WiFi $802.11 \mathrm{n}$ in $2.4 \mathrm{GHz}$ radio band with 20 $\mathrm{MHz}$ channel width, constituted basically of 14 overlapped channels spaced with $5 \mathrm{MHz}$. The channels overlapping is a characteristic used and analyzed in this paper as it will be explained in later sections. Under the same concept, the study presented in this paper could be extended to $5 \mathrm{GHz}$ band with $20 \mathrm{MHz}$ channel width (or with wider channel width e.g. 40 $\mathrm{MHz}$ in channel bonding) or for 802.11ac.

This paper is organized as follows: Section II describes 802.11n physical layer and channel assignment techniques. Our proposed algorithm along with the needed formulation is presented in Section III. In section IV simulation results are shown. Finally, Section V concludes the paper.

\section{System And Channel Assignment Models}

WLAN WiFi is based on IEEE 802.11 standard designed for indoor Wireless Local Area Networks for bandwidths of up to $100 \mathrm{MHz}$, at frequencies of 2 and $5 \mathrm{GHz}$ [7].

The challenge lies when we have more nodes than the available orthogonal channels; therefore, additional numbers of available channels and optimization of the scanning duration for channels assignment are needed due to the existing network load.

In $2.4 \mathrm{GHz}$ band, with $20 \mathrm{MHz}$ channel bandwidth, 802.11n is basically constituted of 14 channels spaced with $5 \mathrm{MHz}$, where the adjacent channels overlap. In Europe, the first 11 channels remain available, and only three channels are non-overlapping in frequency at the same time [2] (e.g. channels 1,5 and 9) as presented in figure 1 .

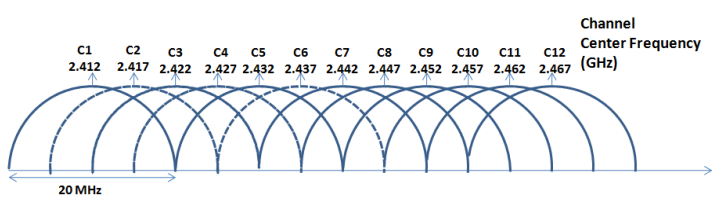

Fig. 1. $\quad 802.11$ n physical overlapped channels

Practically, to avoid the inter channel interference only nonoverlapped channels are used. However, in densely populated networks, and with the constraints of increasing spectrum demand for future WiFi and mobile communication technologies such as $5 \mathrm{G}$, the number of available non-overlapped channels 
may not be enough, thus devices might have to share channels or check for a new spectrum if it becomes available.

For these considerations, we are proposing an algorithm that calculates the load of all channels by observing the distinct 3 non-overlapping channels only (e.g. channels 1, 5 and 9), which assures the measurement of the entire physical channels load.

Note that having the load of each channel facilitates the decision of the user on the channel selection. This decision will be based on the minimal load as a potential criteria to be used when the AP or the user is searching for a suitable channel.

We are proposing in this paper to use the particularity of the channels overlapping in order to measure the load of the overlapped 12 channels through observations done on 3 distinct channels only.

\section{AlgORITHM Formulation}

As we mentioned earlier in this paper, our proposed algorithm is able to estimate the load of the $12 \mathrm{WiFi} 802.11 \mathrm{n}$ channels by performing 3 observations only, and this on the non-overlapped channels, i.e channels 1,5 , and 9.

For the simplification of calculations, and in order to avoid duplications, we are representing here the observation of Channel 1 only. The observation of other channels can be easily generalized by adopting the same concept. Note that by observing Channel 1 , our algorithm is able to estimate the load of channels $1,2,3$ and 4 .

Let us define $\Gamma^{1}(f)$ as the baseband spectrum of the signal observed in channel 1 and $S(f)$ the theoretical baseband Power Spectrum (PS) of the WiFi signal, which emits in a continuous way. According to CSMA/CA principle, Access Points (APs) are not transmitting their data continuously. Let $\alpha_{i}$ denote the load of channel $i$. It is defined as the percentage of the channel $i$ usage in time (or busy time) in respect to the total channel measurement time as described previously.

The observed baseband spectrum of Channel 1 with respect to all signals transmitted in the overlapped channels $i$ is expressed as:

$$
\left(\lambda_{i}^{2}(f) \cdot \alpha_{i}\right) \cdot S(f),
$$

where $\lambda_{i}(f)$ is the signal attenuation due to the propagation model of Channel $i$.

To simplify the presentation of the algorithm, we assume in the following section that the attenuation $\lambda_{i}(f)=1, \forall i, \forall f$; noting that the robustness of the proposed algorithm in presence of a multipath fading channel is shown in the simulation results section.

The observed baseband spectrum $\Gamma^{1}(f)$ can be easily expressed in terms of the theoretical spectrum $S(f)$, which is given by [8]:

$$
S(f)=\frac{\sigma_{c}^{2}}{M T_{s}} \sum_{k=0}^{N-1}\left(\operatorname{sinc}\left[\left(f-k \Delta_{f}\right) M T_{s}\right]\right)^{2},
$$

where $\operatorname{sinc}(\alpha)=\frac{\sin (\pi \alpha)}{\pi \alpha}, \mathrm{M}$ is the symbol length, $\sigma_{c}$ Variance of the data symbols $C_{(k, l)}$ (complex value) modulated on the $k^{t h}$ subcarrier of the $l^{t h}$ symbol, k discrete frequency index, $\mathrm{N}$ number of subcarriers, and $\Delta \mathrm{f}$ the frequency spacing between subcarriers.

The theoretical Power Spectrum Density (PSD) is shown in figure 2 .

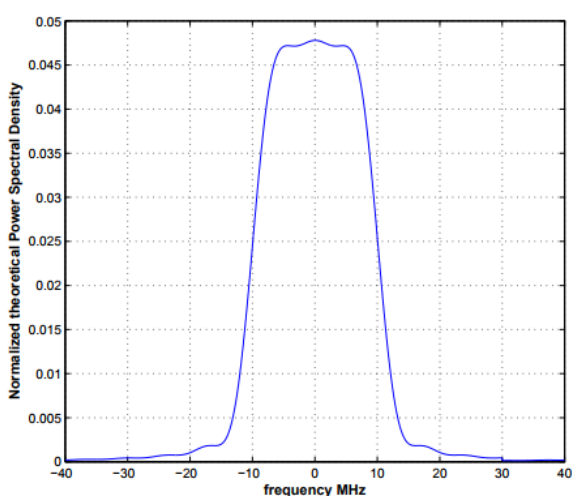

Fig. 2. normalized theoretical Power Spectral Density of the 802.11 n physical channel

To assure the orthogonal relationship between subcarriers, $\Delta \mathrm{f}$ is set as $W / N=1 / M$, where $W$ is the total bandwidth of the signal, and $T_{s}$ is the sampling interval employed in the Orthogonal Frequency Division Multiplexing (OFDM) transmitter.

To estimate $\Gamma^{i}(f)$, we use Welch periodogram method [9]. Mathematically, it is defined as the Fourier transform of the autocorrelation sequence of the time series. This method outlines the application of the Fast Fourier Transform algorithm to the estimation of the power spectra, which involves sectioning the record, taking modified periodograms of these sections, and averaging these modified periodograms [9] [10].

Let us now derive the expression of the power spectrum (PS) $\Gamma^{1}(f)$. Channels $1,2,3$ and 4 contribute to this PS. We are therefore able to estimate the channels load $\alpha_{1}, \alpha_{2}, \alpha_{3}$, and $\alpha_{4}$ from this observation. The contribution of channels 2 , 3 , and 4 in the PS of channel 1 is illustrated in figure 3 after the shifting of the channels to the baseband.

The observation of channel 1 can reflect the total load of channel 1 in addition to a part of the load of its related overlapped channels 2, 3 and 4, according to the overlapped partitions. For a bandwidth B of the channel, the total overlapping bandwidth between two consecutive channels is 3B/4. Based on this sectioning, we divide the theoretical PSD $S(f)$ into 4 partitions $S_{1}, S_{2}, S_{3}$, and $S_{4}$ as per the below and presented in figure 4 :

$$
\begin{aligned}
& S_{1}(f)=S(f) \text { for } f \in[-B / 2 ;-B / 4] \text { and } 0 \text { elsewhere } \\
& S_{2}(f)=S(f) \text { for } f \in[-B / 4 ; 0] \text { and } 0 \text { elsewhere } \\
& S_{3}(f)=S(f) \text { for } f \in[0 ; B / 4] \text { and } 0 \text { elsewhere } \\
& S_{4}(f)=S(f) \text { for } f \in[B / 4 ; B / 2] \text { and } 0 \text { elsewhere }
\end{aligned}
$$


The complete theoretical PSD is the vector: $\mathbb{S}=\left[S_{1}(f), S_{2}(f), S_{3}(f), S_{4}(f)\right]$ of size $(1 \mathrm{x} 4)$. Now let

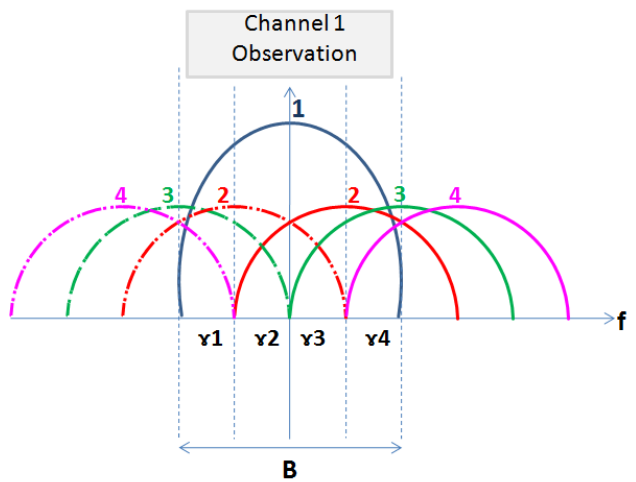

Fig. 3. channel 1 observation model

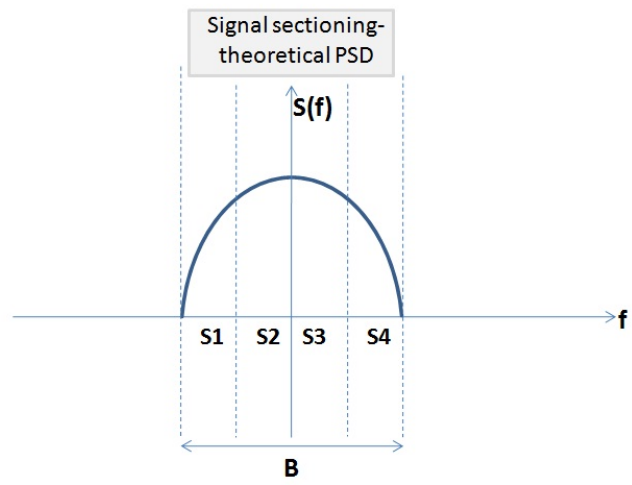

Fig. 4. Signal sectioning - theoretical PSD

$\Gamma^{1}(f)$, which is the PS of the observed signal in channel 1, be divided similarly into 4 sections $\gamma_{1}, \gamma_{2}, \gamma_{3}$, and $\gamma_{4}$ as shown in figure 3 and calculated below:

$\gamma_{1}(f)=\Gamma^{1}(f)$ for $f \in[-B / 2 ;-B / 4]$ and 0 elsewhere

$\gamma_{2}(f)=\Gamma^{1}(f)$ for $f \in[-B / 4 ; 0]$ and 0 elsewhere

$\gamma_{3}(f)=\Gamma^{1}(f)$ for $f \in[0 ; B / 4]$ and 0 elsewhere

$\gamma_{4}(f)=\Gamma^{1}(f)$ for $f \in[B / 4 ; B / 2]$ and 0 elsewhere.

The complete PS is the vector $\Gamma^{1}=$ $\left[\gamma_{1}(f) ; \gamma_{2}(f) ; \gamma_{3}(f) ; \gamma_{4}(f)\right]$ of size $(4 \times 1)$. Based on figure 3 , we need to calculate $\gamma_{1}, \gamma_{2}, \gamma_{3}$, and $\gamma_{4}$ in terms of $S(f)$ and $\alpha_{i}$. We can observe that, since channels $1,2,3$ and 4 shifted to the baseband are duplicated from both sides while saving the same overlapping proportions, $\gamma_{1}$ is constituted of 2 times the load of channel 1 corresponding to section 1 (S1), 1 time the load of channel 2 corresponding to section 2 $(S 2), 1$ time the load of channel 3 corresponding to section $3(S 3)$, and 1 time the load of channel 4 corresponding to section $4(S 4)$. Therefore, we can have the below equation:

$$
\gamma_{1}(f)=2 . \alpha_{1} \cdot S_{1}(f)+\alpha_{2} . S_{2}(f)+\alpha_{3} . S_{3}(f)+\alpha_{4} \cdot S_{4}(f)
$$

By applying the same concept for $\gamma_{2}, \gamma_{3}$ and $\gamma_{4}$, we can write the below equations:

$$
\begin{aligned}
& \gamma_{2}(f)=2 \cdot \alpha_{1} \cdot S_{2}(f)+\alpha_{2} \cdot\left(S_{1}(f)+S_{3}(f)\right)+\alpha_{3} \cdot S_{4}(f) \\
& \gamma_{3}(f)=2 \cdot \alpha_{1} \cdot S_{3}(f)+\alpha_{2} \cdot\left(S_{2}(f)+S_{4}(f)\right)+\alpha_{3} \cdot S_{1}(f) \\
& \gamma_{4}(f)=2 \cdot \alpha_{1} \cdot S_{4}(f)+\alpha_{2} \cdot S_{3}(f)+\alpha_{3} \cdot S_{2}(f)+\alpha_{4} \cdot S_{1}(f)
\end{aligned}
$$

From the above equations, we can write the Power Spectrum of the observed signal in channel 1 as:

$$
\left[\begin{array}{llll}
2 & 0 & 0 & 0 \\
0 & 1 & 0 & 0 \\
0 & 0 & 1 & 0 \\
0 & 0 & 0 & 1 \\
0 & 1 & 0 & 0 \\
2 & 0 & 0 & 0 \\
0 & 1 & 0 & 0 \\
0 & 0 & 1 & 0 \\
0 & 0 & 1 & 0 \\
0 & 1 & 0 & 0 \\
2 & 0 & 0 & 0 \\
0 & 1 & 0 & 0 \\
0 & 0 & 0 & 1 \\
0 & 0 & 1 & 0 \\
0 & 1 & 0 & 0 \\
2 & 0 & 0 & 0
\end{array}\right] \cdot\left[\begin{array}{l}
\alpha_{1} \\
\alpha_{2} \\
\alpha_{3} \\
\alpha_{4}
\end{array}\right]
$$

Now let $\mathbb{B}_{1}$ be equal to:

$$
\mathbb{B}_{1}=\left[\begin{array}{llll}
\mathbb{S} & 0 & 0 & 0 \\
0 & \mathbb{S} & 0 & 0 \\
0 & 0 & \mathbb{S} & 0 \\
0 & 0 & 0 & \mathbb{S}
\end{array}\right] \cdot\left[\begin{array}{llll}
2 & 0 & 0 & 0 \\
0 & 1 & 0 & 0 \\
0 & 0 & 1 & 0 \\
0 & 0 & 0 & 1 \\
0 & 1 & 0 & 0 \\
2 & 0 & 0 & 0 \\
0 & 1 & 0 & 0 \\
0 & 0 & 1 & 0 \\
0 & 0 & 1 & 0 \\
0 & 1 & 0 & 0 \\
2 & 0 & 0 & 0 \\
0 & 1 & 0 & 0 \\
0 & 0 & 0 & 1 \\
0 & 0 & 1 & 0 \\
0 & 1 & 0 & 0 \\
2 & 0 & 0 & 0
\end{array}\right]
$$

then

$$
\Gamma^{1}(f)-\mathbb{B}_{1} \cdot \alpha=0
$$

where $\alpha=\left[\alpha_{1}, \alpha_{2}, \alpha_{3}, \alpha_{4}\right]$ denotes the load of channels 1,2 , 3 and 4.

Our target is now to estimate $\alpha$. Since the channel load has a non-negative value, non-negativity constraint should be applied on the load estimations instead of simple non-square matrix inversion. In this paper, the non-negative Least Mean Square (LMS) calculation has been applied. It is derived based on a stochastic gradient descent approach [11] combined with a fixed-point iteration strategy that ensures convergence toward a solution to estimate vector $\alpha$ from Channel 1 . 
We denote by $\left[\hat{\alpha}_{1}^{1}, \hat{\alpha}_{2}^{1}, \hat{\alpha}_{3}^{1}, \hat{\alpha}_{4}^{1}\right]$ the estimate of the load of channels 1, 2, 3 and 4 obtained from the observation of Channel 1. It is given by:

$$
\left[\begin{array}{c}
\hat{\alpha}_{1}^{1} \\
\hat{\alpha}_{2}^{1} \\
\hat{\alpha}_{3}^{1} \\
\hat{\alpha}_{4}^{1}
\end{array}\right]=\operatorname{Argmin}_{\alpha}\left(\left\|\hat{\Gamma}^{1}(f)-\mathbb{B}_{1} \cdot \alpha\right\|\right)
$$

We proceed similarly for the remaining 2 non-overlapped channels 5 and 9 in order to recover the load of the 12 channels as per the below equations, noting by $\hat{\Gamma}^{j}(f)$ as the estimated baseband spectrum of the signal of channel $j$ :

$$
\hat{\Gamma}^{5}(f)=\mathbb{B}_{5} \cdot\left[\begin{array}{c}
\hat{\alpha}_{2}^{5} \\
\hat{\alpha}_{3}^{5} \\
\hat{\alpha}_{4}^{5} \\
\hat{\alpha}_{5}^{5} \\
\hat{\alpha}_{6}^{5} \\
\hat{\alpha}_{7}^{5} \\
\hat{\alpha}_{8}^{5}
\end{array}\right]
$$

and

$$
\hat{\Gamma}^{9}(f)=\mathbb{B}_{9} \cdot\left[\begin{array}{c}
\hat{\alpha}_{6}^{9} \\
\hat{\alpha}_{7}^{9} \\
\hat{\alpha}_{8}^{9} \\
\hat{\alpha}_{9}^{9} \\
\hat{\alpha}_{10}^{9} \\
\hat{\alpha}_{11}^{9} \\
\hat{\alpha}_{12}^{9}
\end{array}\right]
$$

Therefore from equation (7) we are able to estimate the load of channels 5, 6, 7 and 8 and from equation (8) we are able to estimate the load of channels 9, 10, 11 and 12 .

\section{Simulation Results}

A simulation using Matlab has been developed to generate the physical signal of $802.11 \mathrm{n}$ based on the Orthogonal Frequency Division Multiplexing (OFDM) technique as per basic 802.11n transmitter, and according to WiFi 802.11n related parameters shown in Table I.

TABLE I

USED $802.11 \mathrm{~N}$ PARAMETERS

\begin{tabular}{|l|l|}
\hline Parameters & Values \\
\hline Bandwidth & $20 \mathrm{MHz}$ \\
\hline $\begin{array}{l}\text { The frequency spacing be- } \\
\text { tween subcarriers }\end{array}$ & $312.5 \mathrm{Khz}$ \\
\hline $\begin{array}{l}\text { Sampling interval } \\
\text { employed in the OFDM } \\
\text { transmitter Ts }\end{array}$ & $0.05 \mu \mathrm{s}$ \\
\hline Symbol length & $3.2 \mu \mathrm{s}$ \\
\hline Number of subcarriers & 64 \\
\hline FFT Window & 64 \\
\hline Modulation & $16 \mathrm{QAM}$ \\
\hline $\begin{array}{l}\text { Total number of samples } \\
\text { per OFDM symbol }\end{array}$ & 1024 \\
\hline $\begin{array}{l}\text { Number of samples zero- } \\
\text { padded after 16 QAM }\end{array}$ & 2048 \\
\hline Number of symbols & 200 \\
\hline
\end{tabular}

The length of the input signal used in our simulation is equivalent to the duration of 200 OFDM symbols in time (or 200 times the symbol duration $\left(t_{s}\right)=3.2 \mu \mathrm{s}$ ), where the channel load is expressed by non-zero symbols value equivalent to the time occupation of the signal (or busy time), and with null symbols value when the channel is empty (or idle time).

The channels load predefined on the twelve channels is expressed as the percentage of the channel occupation time between $0 \%$ and $100 \%$ (or 0 and 1) assumed as following: $20 \%, 50 \%, 10 \%, 40 \%, 90 \%, 40 \%, 60 \%, 70 \%, 80 \%, 40 \%$, $30 \%, 90 \%$.

As explained previously, since the physical channels overlap with only 3 distinct channels, an observation of those 3 distinct channels entails to measure the load of the 12 channels. Therefore, we start first by observing channels 1 , 5 , and 9. By applying the method presented in the previous section, the load of channels $1,2,3$, and 4 is estimated from the observation of channel 1 , the load of channels $5,6,7$ and 8 is estimated from the observation of channel 5, and the load of channels $9,10,11$ and 12 is estimated from the observation of channel 9.

As shown in figure 5, the estimated load is nearly the same comparing to the predefined load.

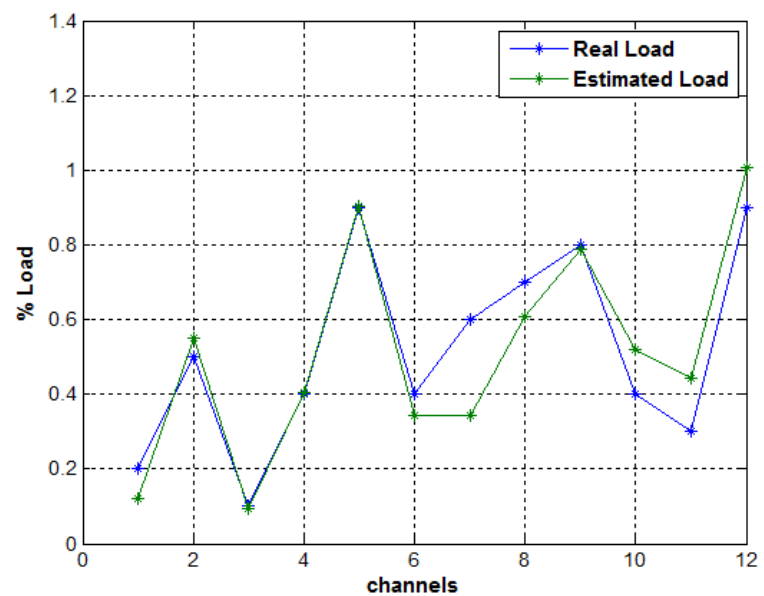

Fig. 5. Estimated load versus the real load with 3 channels observation.

We assume now that the channel is affected by a White Gaussian Noise. In order to analyze the noise effect on the accuracy of our algorithm, same observations are used to reflect the estimated load versus the real one. The MSE value is represented in respect to Signal to Noise Ratio (SNR) in figure 6 as the average of repetitive simulations.

We can notice that the precision of the algorithm is affected by a high noise level; however an acceptable error margin can still exist with a SNR around $3 \mathrm{~dB}$.

Finally, following the assumption that the attenuation is not affecting our calculations $\left(\lambda_{i}(f)=1, \forall i, \forall f\right)$, non-perfect conditions are assumed now in the presence of a multipath 


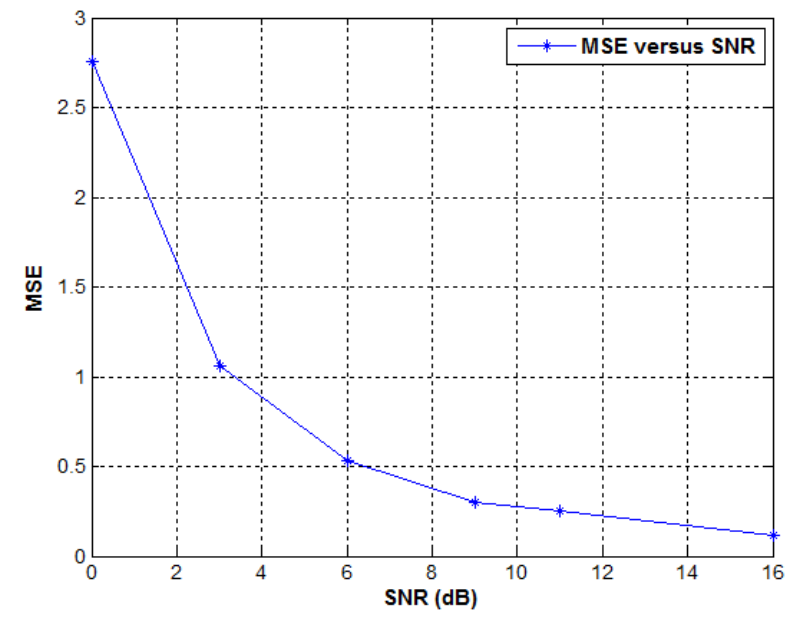

Fig. 6. Averaged Mean Squared Error of the estimated load versus Signal to Noise Ratio values.

fading channels.

Our simulated OFDM signal has been filtered through a normalized multipath fading channel to reflect the effective Power Spectral Density and thus calculate the channels load as previously explained in this paper.

We can observe in figure 7 that our algorithm is still constantly accurate despite certain attenuation factors.

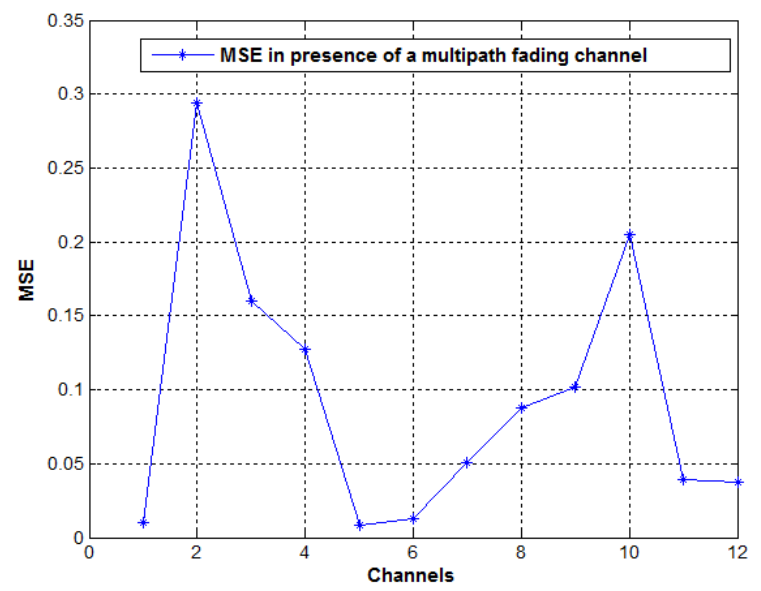

Fig. 7. Averaged Mean Squared Error of the estimated load versus the real load values in presence of a multipath fading channel.

\section{CONCLUSION}

In this paper we have proposed an algorithm that estimates the load of the physical channels of WiFi $802.11 \mathrm{n}$ in $2.5 \mathrm{GHz}$ spectrum. Based on only 3 observations of non overlapping channels, the proposed algorithm is able to estimate the load of the 12 channels of the WiFi 802.11n.

The accuracy of the algorithm has been measured by the Mean Squared Error of multiple realizations, in error free channel and in white gaussian noisy channel. We evaluated our work and can conclude that the proposed algorithm has a high accuracy level and flexibility in estimating the load of the physical channels, thus facilitating the channel assignment based on the minimal load and providing better Quality of Experience (QoE) for the WiFi terminal. Following the same principle, the analysis of $5 \mathrm{GHz}$ spectrum and 802.11ac could be applied.

\section{REFERENCES}

[1] Zhu Han, Thanongsak Himsoon, Wipawee Pam Siriwongpairat, and K J Ray Liu, Resource Allocation for Multiuser Cooperative OFDM Networks: Who Helps Whom and How to Cooperate, IEEE Transactions On Vehicular Technology, 58, 2378-2391 (2009)

[2] Amit Kachroo, Junhyuk Park and HyungWon Kim, Channel Assignment with Transmission Power Optimization method for High throughput in Multi-Access Point WLAN, IEEE, ICT and Future Planning as Global Frontier Project (CISS-2014011066) (2015)

[3] S. Chieochan, E. Hossain and J. Diamond, Channel Assignment Schemes for Infrastructure based WLANs, IEEE Communications Surveys and Tutorial, 12, 124136 (2010)

[4] Dawei Gong, Miao Zhao and Yuanyuan Yang, Channel Assignment in Multi-Rate 802.11n WLANs, IEEE Wireless Communications and Networking Conference (2013)

[5] Minho Kim and Chong-Ho Choi, Hidden-Node Detection in IEEE 802.11n Wireless LANs, IEEE Transactions On Vehicular Technology, 62 (2013)

[6] Nicolas Montavont, Andres Arcia Moret and German Castignani, On the Selection of Scanning Parameters in IEEE 802.11 Networks, IEEE 24th International Symposium on Personal, Indoor and Mobile Radio Communications: Mobile and Wireless Networks, 2137-2141 (2013)

[7] Rachana Khanduri, S. S. Rattan, Performance Comparison Analysis between IEEE $802.11 \mathrm{a} / \mathrm{b} / \mathrm{g} / \mathrm{n}$ Standards, International Journal of Computer Applications, 78, 09758887 (2013)

[8] Toon van Waterschoot and Vincent Le Nir and Jonathan Duplicy and Marc Moonen, Analytical Expressions for the Power Spectral Density of CP-OFDM and ZP-OFDM Signals, IEEE Signal Processing Letters, 17, 1070-9908 (2010)

[9] Peter D.Welch, The Use of Fast Fourier Transform for the Estimation of Power Spectra: A Method Based on Time Averaging Over Short, Modified Periodograms, IEEE Trans. Audio and Electroacoust., 15, 7073 (1967)

[10] Hansa Rani Gupta, Rajesh Mehra, Power Spectrum Estimation using Welch Method for various Window Techniques, International Journal of Scientific Research Engineering and Technology (IJSRET), 2, 389392 (2013)

[11] Jie Chen, Cedric Richard, Jose Carlos M Bermudez, and Paul Honeine, Nonnegative least-mean square algorithm, IEEE Transactions on Signal Processing, 59, 5225-5235 (2011) 